\title{
FECUNDANDO O PROCESSO DA INTERDISCIPLINARIDADE NA INICIAÇÃO CIENTÍFICA
}

\author{
Luciano Mazon ${ }^{1}$ \\ Maria Auxiliadora Trevizan ${ }^{2}$
}

Mazon L, Trevizan MA. Fecundando o processo da interdisciplinaridade na iniciação cientifica. Rev Latino-am Enfermagem 2001 julho; 9(4):837.

O enfoque disciplinar moderno fundamenta-se em processos especificos, apresentando uma inclinação reducionista do conhecimento como conseqüência da postura cientifica altamente racional que promoveu a excessiva especialização e fragmentação, resultando em depauperamento do ser humano. Com o desenvolvimento das sociedades tendo em perspectiva a interconexão do conhecimento, buscando cada vez mais a instituição de dimensões mais ampliativas e globalizantes nas esferas do saber e do fazer, a tendência da disciplinaridade é ser transcendida. O objetivo deste trabalho é relatar experiência em programa de iniciação científica sob a ótica da interdisciplinaridade. No desempenho de papéis de orientado e orientador de áreas distintas - Administração e Enfermagem - estamos vivenciando o processo da interdisciplinaridade através do intercâmbio de idéias, ações e de investigação sobre recursos humanos e seu gerenciamento no campo da Enfermagem. No nosso entender, sua disseminação poderá contribuir para o oferecimento de subsídios capazes de fortalecer a interdisciplinaridade já no contexto da iniciação científica.

PALAVRAS CHAVE: administração, enfermagem, educação

\section{STRENGTHENING THE INTERDISCIPLINARY PROCESS IN UNDERGRADUATE STUDENTS' RESEARCH PROGRAMS}

The modern disciplinary focus is based on specific processes and presents a tendency to reduce knowledge by describing it as a consequence of a highly rational scientific posture that has promoted excessive specialization and fragmentation in detriment of the human being. With the development of societies and the perspective of knowledge interconnection which searches for institutions with global and broad dimensions in the spheres of knowing and doing, disciplinarity will be transcended. In this sense, the purpose of this work is to report the experience of an undergraduate students' research program in the view of interdisciplinarity. Playing the roles of advisor and student from different areas - Nursing and Administration respectively - we are experiencing an interdisciplinary process through the exchange of ideas, actions and the investigation on human resources and their management in the Nursing area. We believe that the dissemination of this experience can contribute to strengthen interdisciplinarity in the context of research in undergraduation programs.

KEY WORDS: administration, nursing, education

\section{FECUNDANDO EL PROCESO DE LA INTERDISCIPLINARIEDAD EN LA INICIACIÓN CIENTÍFICA}

El enfoque disciplinar moderno se fundamenta en procesos específicos presentando una inclinación reduccionista del conocimiento como consecuencia de la postura científica racional que promovió la excesiva especialización y fragmentación, trayendo como resultado el desprecio por el ser humano. Con el desarrollo de las sociedades y teniendo como perspectiva la interconexión del conocimiento, buscando cada vez más la institución de dimensiones más amplias y globales en las esferas del saber y del hacer, la tendencia de la disciplinariedad es la de ser transcendida. El objetivo de este trabajo es relatar ésta experiencia en un programa de iniciación cientíica desde la óptica de la interdisciplinariedad. En el desempeño de los papeles de alumno y orientador de áreas distintas - Administración y Enfermería - estamos viviendo el proceso de interdisciplinariedad a través del intercambio de ideas, acciones y de investigación sobre recursos humanos y su gerencia en el campo de la Enfermería. Creemos que su diseminación podrá contribuir ofreciendo aportes capaces de fortalecer la interdisciplinaridad en el contexto de la iniciación científica.

PALABRAS CLAVES: administración, enfermería, educación

\footnotetext{
${ }^{1}$ Aluno de graduação em Administração da Faculdade de Economia, Administração e Contabilidade da Universidade de São Paulo - Campus de Ribeirão Preto, Bolsista do CNPq, vinculado ao Projeto "Capital Humano da Enfermagem no meio Hospitalar"sob a responsabilidade da Profa Dra Maria Auxiliadora Trevizan; ${ }^{2}$ Pesquisador $1 \mathrm{~A}$ do CNPq, Professor Titular da Escola de Enfermagem de Ribeirão Preto da Universidade de São Paulo, Centro Colaborador da OMS para o desenvolvimento da pesquisa em enfermagem, e-mail: trevizan@eerp.usp.br
} 
INTRODUÇÃOO

A disciplinaridade se alicerça na visão analítica que privilegia a decomposição do todo em partes, fundamentada em processos específicos, racionais e sensoriais, apresentando uma inclinação reducionista do conhecimento. 0 enfoque disciplinar moderno é conseqüência essencial da postura científica altamente racional e rigorosa que promoveu a excessiva especialização e fragmentação, resultando em alienação e depauperamento do ser humano.

Destaca-se 0 limite de percepção que obscurece a visão do todo provocada pela exagerada especialização. Mesmo com seu importante papel, a ciência contém em si uma característica de artificialidade, acarretando dois limites: " a) não tem da realidade um olhar abrangente, mas tendencialmente recortado em partes; b) a especialização é sua tática de aprofundamento analítico, mas não dá, sozinha, conta de uma realidade complexa"(1).

Precisamos transcender este enfoque.

Neste sentido, a transdisciplinaridade é uma evolução qualitativa, alicerçada no método sintético, enfatizando a totalidade real, apresentando, portanto, uma postura ampliativa. $\mathrm{Na}$ Universidade Holística Internacional de Brasília, a transdisciplinaridade é definida como " o encontro de várias áreas do conhecimento em torno de uma axiomática comum, ou princípios comuns subjacentes" ${ }^{\prime 2)}$.

Weil ${ }^{(3)}$ argumenta que "o encontro interdisciplinar, entendido como interação ou síntese entre duas ou várias disciplinas, favorece a emergência da transdisciplinaridade". 0 enfoque interdisciplinar, impulsionado pelo movimento holístico, tem em perspectiva integrar em conjuntos cada vez mais abrangentes o que foi fragmentado, decomposto pelo homem, traduz a mobilização pela correlação entre as disciplinas ${ }^{(3)}$.

Interdisciplinaridade é definida como "a arte do aprofundamento com sentido de abrangência, para dar conta, ao mesmo tempo, da particularidade e da complexidade do real"(1). Devido a suas implicações, a interdisciplinaridade é melhor praticada em grupo, agregando a contribuição qualitativa das especialidades, num esforço de reconstrução do conhecimento, com vistas à inovação ${ }^{(1)}$. Assim, a compreensão da necessidade de inovar e o desenvolvimento da inovação se realizam mais facilmente, e melhor, em ambiente interdisciplinar. Ao tratar do desafio de inovar, este autor argumenta que para o entendimento da complexidade do real tornase imperioso "a formação mais abrangente do pesquisador, sobretudo em termos de métodos comuns e trabalho em equipe, para permitir o concurso orquestrado dos especialistas e a aprendizagem coletiva e intersubjetivamente crítica"(1).
No contexto universitário convivem todas as áreas do conhecimento. Entretanto, a dimensão fundamental da universidade - que é "a convivência das múltiplas expressões do saber" - acaba sendo fragmentada pela própria estrutura universitária, dificultando o convívio na diversidade ${ }^{(4)}$.

Ao abordar sobre a missão acadêmica, referido autor ${ }^{(4)}$ atribui à universidade o papel de formação da cidadania, salientando que, possivelmente, sua função principal seja "desenvolver a inquietude do ser social". Em sua busca de renovação, recomenda que "uma nova universidade deve ser integrada, empreendedora e generosa". É integrada na medida em que fomenta a melhor utilização de recursos inter e intradepartamental, e quando promove 0 intercâmbio e o estreitamento de relações e vínculos com outras instituições, favorecendo o avanço do conhecimento, através da atuação de recursos humanos em atmosfera interdiciplinar, de valorização da diversidade e de mobilização transdisciplinar. É generosa quando "integra conhecimentos para consolidar competência, e sempre a serviço do interesse coletivo". É empreendedora quando antecipa-se ao futuro com competência, cultivando o sentido de responsabilidade e de inovação( ${ }^{(4)}$.

Neste sentido, a pesquisa e o desenvolvimento científico encontram na universidade seu habitat por excelência. No entender de Mendes ${ }^{(5)}$, "uma das grandes perspectivas da pesquisa desenvolvida no meio universitário consiste exatamente em poder voltar-se para as reais necessidades da sociedade brasileira como um todo... uma pesquisa de cunho altamente comunitário, visando ao bem estar de todos e à melhoria da qualidade de vida". Compreendemos que subjaz a este posicionamento um envolvimento de pesquisa interdisciplinar, uma vez que a interconexão do conhecimento busca cada vez mais a instituição de dimensões mais ampliativas e globalizantes nas esferas do saber e do fazer.

A Enfermagem e a Administração, por sua própria natureza, necessitam da interdisciplinaridade para o seu envolvimento na transdisciplinaridade.

Das considerações expostas, o objetivo deste trabalho é relatar experiência de iniciação científica sob a ótica da interdisciplinaridade. No nosso entender, sua disseminação poderá contribuir para o oferecimento de subsídios capazes de fortalecer a interdisciplinaridade já no contexto da iniciação científica.

\section{RELATO DE EXPERIÊNCIA EM PROJETO DE INICIAÇÃO CIENTÍFICA}

Em nosso mundo contemporâneo, cuja composição se dá pela chamada sociedade da informação, estamos cada vez mais 
buscando novos conhecimentos para nos adequar a este contexto, objetivando puramente a realização pessoal ou visando melhores oportunidades no competitivo mercado de trabalho.

Há defensores da idéia de que há necessidade de especialização em certas áreas como um diferencial para o mercado; porém, não podemos nos alienar frente aos conhecimentos que estão diretamente relacionados com o que buscamos, cujos conteúdos fornecem dados que facilitarão nossa adaptação a novos ambientes.

A interdisciplinaridade, como referimos, corresponde a toda atividade vivenciada a partir de diferentes enfoques; abarca um determinado fenômeno que deve ser estudado por vários e diferentes ramos de conhecimento, focando-o como um todo, ou seja, é a integração de diversos conteúdos que se convergem para certo objetivo específico.

No momento em que estamos dedicando a maior parte de nosso tempo aos estudos, temos oportunidade de envolvermo-nos em meios que não estão diretamente relacionados com a carreira profissional que escolhemos. Além de estarmos ampliando o nosso leque cultural, estamos agregando conhecimentos que servirão de apoio, ou até mesmo orientação, em nossa futura profissão.

Como estudante de administração de empresas da FEARP/ USP*, percebemos a oportunidade de intercâmbio com uma outra unidade do campus de Ribeirão Preto, caracterizado por um forte potencial biológico. Essa oportunidade foi encontrada na EERP/ USP $^{* *}$, onde passamos a conviver com uma nova fase no processo ensino-aprendizagem: interdisciplinaridade entre as ciências exatas, biológicas e humanas.

Se focarmos os dois cursos em si, administração e enfermagem, à princípio poderá parecer que são tênues as ligações entre essas duas disciplinas, já que uma trata de números, gráficos, aplicações financeiras, cálculos, produtos, preços, indústrias, mercados, economias, através de profissionais predominantemente do sexo masculino, e a outra está centrada em pacientes, medicamentos, hospitais, cuidados, tratamentos, promoção de saúde, recuperação de doença e seus profissionais são preponderantemente do sexo feminino.

Porém, ampliando nossa visão, o que poder-se-ia dizer de liderança, recursos humanos, comunicação, trabalho em equipe, administração de estoque de medicamentos, planejamento, organização, direção e controle?
Em qual das duas disciplinas que esses itens estão relacionados?

Com a nossa integração, em 1998, como bolsista de iniciação científica ao projeto "Capital Humano da Enfermagem no meio hospitalar: enfoque sobre a liderança do enfermeiro", subvencionado pelo CNPq (Conselho Nacional de Desenvolvimento Científico e Tecnológico), em desenvolvimento no GEPURHEn ${ }^{* * *}$, passamos a vivenciar o processo da interdisciplinaridade através do intercâmbio de idéias e de investigação sobre recursos humanos e seu gerenciamento no campo da enfermagem.

No início de nossa participação nesse projeto foram desenvolvidos programas de estudos sobre motivação, liderança, comportamento humano no trabalho, gestão hospitalar, recursos humanos, gerenciamento, inovação, terceiro setor, comunicação, burocracia, entre outros, relacionados à enfermagem e à administração, tendo como objetivo aprofundar conhecimento sobre a inter-relação existente entre ambas as áreas, redundando no desenvolvimento de um trabalho científico.

0 trabalho desenvolvido ${ }^{(6)}$, abordou uma experiência administrativa realizada em um hospital psiquiátrico. Trata-se de uma instituição filantrópica que presta assistência a pacientes com problemas mentais, bem como a farmacodependentes. Apresentava na ocasião, um alto "turnover" de pessoal, o que prejudicava o desenvolvimento das atividades no âmbito da organização e em conseqüência a melhor assistência aos clientes. Assim sendo, integramos uma equipe que realizou o processo de recrutamento $\mathrm{e}$ seleção de pessoal para diversos setores da instituição, com o acompanhamento dos respectivos responsáveis. Após esta intervenção, elaboramos o relato desta experiência. Denominado "Recrutamento e seleção de recursos humanos em um hospital psiquiátrico de um município paulista", o estudo foi apresentado no $6^{\circ}$ SICUSP (Simpósio de Iniciação Científica da USP), em novembro de 1998 no campus de Ribeirão Preto, foi publicado na Revista LatinoAmericana de Enfermagem ${ }^{(6)}$, e já aprovado para apresentação em mais dois congressos ${ }^{* * * *}$.

Ainda como estagiário do referido hospital desenvolvemos as seguintes atividades: a)administrativas - vinculadas à organização, métodos e informatização dos processos de trabalho, com a criação de um novo organograma; b) de cunho financeiro - reestruturação contábil e renegociação de dívidas anteriores; c) marketing -

\footnotetext{
* FEARP/USP - Faculdade de Economia, Administração e Contabilidade da Universidade de São Paulo - Campus Ribeirão Preto ${ }^{* *}$ EERP/USP - Escola de Enfermagem de Ribeirão Preto da Universidade de São Paulo

*** GEPURHEn - Grupo de Estudos e Pesquisas sobre Utilização de Recursos Humanos em Enfermagem, criado em 1987, pela Profa ${ }^{a}{ }^{a}$ Maria Auxiliadora Trevizan na EERP/USP e por ela coordenado desde então. Cadastrado no Diretório de Grupos de Pesquisa do Brasil - CNPq desde a versão 1.0.

**** IV SEMEAD (Seminário de Administração de Empresas da USP - campus de São Paulo) e no SEAC (Seminário de Administração, Economia e Contabilidade do campus de Ribeirão Preto)
} 
divulgação da instituição através de revistas, jornais, televisão, simpósio de iniciação científica, etc; e d) de recursos humanos legalização de toda parte de pessoal, planejamento de recursos humanos, descrição de cargos e funções e interação da administração com todos os setores do hospital. 0 trabalho na área de recursos humanos foi o que mais obteve benefícios oriundos da interdisciplinaridade.

Outras experiências desenvolvidas relacionam-se ao chamado Terceiro Setor, que são organizações privadas em sua forma, porém, que possuem propósitos públicos. São iniciativas que não visam ao lucro, mantidas pelo Estado e/ou por trabalhos voluntários realizando atividades na esfera pública visando o interesse e a melhoria da sociedade.

O início de nossa atuação nessa área foi a de membro fundador do GEATS - Grupo de Estudo e Apoio do Terceiro Setor vinculado à FEARP/USP, que tem como objetivo "prover o intercâmbio entre Universidade e a Sociedade para fomentar discussões sobre a realidade das organizações sem fins lucrativos em Ribeirão Preto". Com a composição desse grupo, participamos como membro organizador do Fórum do Terceiro Setor, promovido pela FEARP/ USP e pela Fundação SOBECCan - Hospital do Câncer de Ribeirão Preto, em março de 1999. O Fórum teve como objetivo discutir o Terceiro Setor na região de Ribeirão Preto, sua realidade, perspectivas e possibilidades de crescimento e parcerias. Com o acompanhamento, interação e envolvimento nessa área, passamos a desenvolver um estudo intitulado "Análise Comparativa das Instituições do Terceiro Setor de Ribeirão Preto em relação aos Estados Unidos", país que se apresentado no mais alto desenvolvimento nesse setor. É uma pesquisa que ainda se encontra em desenvolvimento.

Também em decorrência da iniciação científica foi a rica experiência de participação na organização de evento internacional na qualidade de Membro de Apoio da Comissão Organizadora do VI Colóquio Pan-Americano de Investigação em Enfermagem, realizado na Escola de Enfermagem de Ribeirão Preto - USP, em maio de 1998. Atuamos como monitor social e interprete, oferecendo apoio logístico. Os participantes conferencistas eram procedentes dos Estados Unidos, Canadá, Argentina, Colômbia, México, Espanha, França, Inglaterra, Coréia do Sul, etc. Durante a realização do Colóquio tivemos a oportunidade de ouvir a respeito do processo de pesquisa na enfermagem, especificamente, sobre investigação em recursos humanos, pesquisa inter e transdisciplinar, bem como conferências sobre a questão saúde no limiar da era pós-moderna. Mantivemos contato e intercâmbio de idéias com enfermeiras brasileiras e estrangeiras a respeito da administração em enfermagem e da importância da liderança nessa profissão. Para nós representou um momento de rica aprendizagem na organização de um evento internacional de grande porte.

Vivenciamos também a experiência de desenvolver pesquisa com aluno de pós-graduação em Enfermagem Fundamental, nível mestrado, articulada pela orientadora comum e também com a participação de outra doutora em enfermagem integrante do GEPURHEn. Intitulada "(In) satisfação da enfermeira na sua condução gerencial", com o objetivo de verificar os fatores que têm fundamentado a (in)satisfação da enfermeira ao exercer 0 gerenciamento, a pesquisa teve como referencial teórico os pressupostos de Maslow e Herzberg. Seus resultados foram apresentados no $51^{\circ}$ Congresso Brasileiro de Enfermagem e 10 Congreso Panamericano de Enfermeria, realizados em Florianópolis, em outubro de 1999. Este trabalho também foi aprovado para ser apresentado no $11^{\text {th }}$ International Congress on Women's Health Issues, a ser realizado em São Francisco, Califórnia - USA.

\section{CONSIDERAÇÕES FINAIS}

Acreditamos que 0 empreendimento de esforços para 0 desenvolvimento do conhecimento através da pesquisa deve ser iniciado no curso de graduação. Desta forma, o estudante estará se concientizando da importância do processo de investigação, bem como da comunicação de seus resultados e da sua utilização ${ }^{(7)}$.

Neste sentido, o CNPq conceitua a iniciação científica como um instrumento que possibilita a introdução do estudante de graduação com o potencial mais promissor na investigação científica, através de sua vinculação a um projeto integrado. Trata-se de um instrumento básico de formação, que tem em perspectiva 0 treinamento em metodologia científica, o desenvolvimento da análise e do julgamento crítico, e o incentivo à criatividade e à inovação.

No trabalho que ora apresentamos focalizamos uma experiência de iniciação científica vinculada à interdisciplinaridade. Esta vinculação permite a construção ou reconstrução do conhecimento, através da ação conjunta de profissionais de diferentes áreas, possibilitando a desenvoltura de integração dos especialistas.

Consideramos ainda que o trabalho interdisciplinar favorece a maior e melhor nitidez da visibilidade da Enfermagem para outros profissionais. Desta forma, recomendamos que este processo seja fecundado já na iniciação científica. 


\section{REFERÊNCIAS BIBLIOGRÁFICAS}

1. Demo P. - Conhecimento moderno: sobre ética e intervenção do conhecimento. Petrópolis (RJ): Vozes; 1997 p. 85, 88, 143.

2. Crema R. - Além das Disciplinas: reflexões sobre transdisciplinaridade geral. In: Weil P, Dámbrosio U, Crema R. Rumo à nova transdisciplinaridade: sistemas abertos de conhecimento. $3^{\mathrm{a}}$ ed. São Paulo (SP): Summus; 1993. p. 132.

3. Weil P. - Axiomática transdisciplinar para um novo paradigma holístico. In: Weil P, Dámbrosio U, Crema R. Rumo à nova transdisciplinaridade: sistemas abertos de conhecimento. $3^{\mathrm{a}}$ ed. São Paulo (SP): Summus; 1993. p. 35, 29.
4. Marcovitch J. A universidade (im)possível. São Paulo (SP): Futura; 1998. p.23, 175, 176.

5. Mendes IAC. - Pesquisa em enfermagem: impacto na prática. São Paulo (SP): Edusp; 1991. p.17.

6. Mazon L, Trevizan MA. Recrutamento e seleção de recursos humanos em um hospital psiquiátrico de um município paulista. Rev Latino-am Enfermagem 2000 agosto; 8(4):81-7.

7. Trevizan MA, Mendes IAC. Iniciação científica: modalidade de incentivo à pesquisa em enfermagem. Rev Gaúch Enfermagem 1991 julho; 12(2):33-38. 